\title{
Suprarrenalectomía abierta y laparoscópica. Revisión de 10 años
}

\author{
Aguilera Bazán A, Pérez Utrilla M, Alonso y Gregorio S, Cansino Alcaide R, Cisneros Ledo J, \\ De la Peña Barthel J.
}

Servicio de Urología. Hospital Universitario La Paz. Madrid.

Actas Urol Esp. 2006;30(10):1025-1030

\section{RESUMEN}

SUPRARRENALECTOMÍA ABIERTA Y LAPAROSCÓPICA. REVISIÓN DE 10 AÑOS

Presentamos los resultados de la revisión de los 10 últimos años de suprarrenalectomías abiertas realizadas en nuestro Servicio, así como el impacto de la suprarrenalectomía laparoscópica introducida en el último año dentro del programa de cirugía laparoscópica retroperitoneal del Hospital Universitario La Paz. La primera adrenalectomía laparoscópica se realizó tras 21 intervenciones retroperitoneales. La experiencia inicial ha sido lo suficientemente buena como para reducir progresivamente las contraindicaciones y aumentar el número de cirugias realizadas con dicha técnica.

Palabras clave: Adrenalectomía. Cirugía endocrinológica. Carcinoma suprarrenal. Feocromocitoma. Laparoscopia.

\section{ABSTRACT}

OPEN AND LAPAROSCOPIC ADRENALECTOMY. 10 YEARS REVIEW

We present a 10 years open adrenalectomy review in our Service and the beginning of laparoscopic adrenalectomy in the last year as a part of the retroperitoneal laparoscopic program at the Hospital Universitario La Paz . The first laparoscopic adrenalectomy was done after 21 retroperitoneal laparoscopic surgeries. Our initial experience has been so good that we have reduced the contraindications for this technique and we have increased the number of laparoscopic surgery cases.

Keywords: Adrenalectomy. Endocrine surgery. Adrenal carcinoma, Pheochromocytoma. Laparoscopy.

$\mathrm{L}^{2}$ a patología benigna o maligna de la glándula suprarrenal no es excesivamente frecuente y por lo general se trata de un campo estudiado por diversas especialidades (Endocrinología, Nefrología, Medicina Interna). Si añadimos que el territorio quirúrgico de dicha glándula es un campo con frecuencia compartido con los cirujanos generales, tendremos otro factor más que nos explique la "fuga" de pacientes con patología quirúrgica suprarrenal hacia otros especialistas ${ }^{1}$.

Como ejemplo de esta dispersión en el tratamiento del paciente con patología endocrinológica quirúrgica en general (tiroides, paratiroides y adrenal), Saunders et al. se hacen esta pregunta “¿Quién realiza la cirugía endocrinológica en Estados Unidos? ${ }^{2}$. El estudio, que abarca desde 1988 hasta 2000, se basa en los datos recogidos por la NIS (National Inpatient Sample) y aunque tiene sus limitaciones en la recogida de datos, refleja lo poco frecuente de la patololgía suprarrenal y lo distribuida que se encuentra su resolución quirúrgica (el 94\% de las 4.209 suprarrenalectomías recogidas estarían realizadas por cirujanos de diferentes especialidades cuya actividad anual de cirugía endocrinológica supone menos del $25 \%$ de sus intervenciones quirúrgicas).

Desde que en 1926 Cesar Roux (Suiza) y Charles Mayo (USA) realizaran la primera cirugia para tratamiento de la patología adrenal, en concreto una adrenalectomía unilateral por feocromocitoma $^{3}$, la técnica de extirpación de dicha glándula ha experimentado diversas modificaciones, buscando generalmente una mejor exposición de 
la glándula y una disminución de la morbilidad de dicha cirugía. La técnica de abordaje inicialmente elegida fue la vía anterior, lo que permitía la exploración de la cavidad abdominal o de la otra glándula suprarrenal en el caso de que la patología adrenal fuera un feocromocitoma. Estos abordajes anteriores podían realizarse mediante una incisión subcostal bilateral (Chevron) (Fig. 1) o una laparotomía media. La vía posterior, a priori con una menor morbilidad, quedó reservada para tumores de menor tamaño. Este abordaje implicaba un campo visual más limitado que la vía anterior y como gran ventaja suponía una rápida identificación de la vena suprarrenal derecha, generalmente más difícil por vía anterior. El abordaje toraco-abdominal descrito principalmente para grandes masas suprarrenales derechas, implica una incisión desde la $9^{\mathrm{a}}$ o $10^{\mathrm{a}}$ costillas con apertura a través del diafragma y extensión intraperitoneal y permite un mejor control de la cava cuando ésta pueda estar afectada por el tumor, pero supone una elevada tasa de complicaciones.

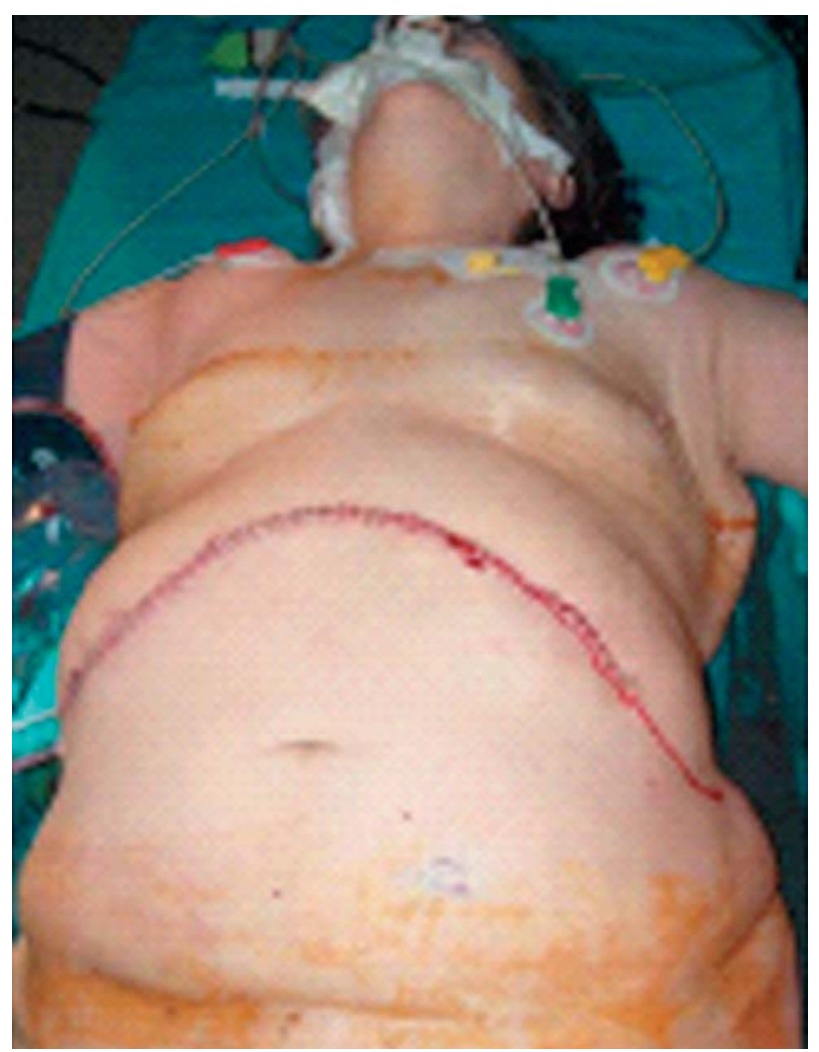

FIGURA 1. Incisión subcostal bilateral.
La morbilidad de todas estas incisiones varía según los diversos autores, $40 \%$ en un artículo publicado en 2005 por el grupo de Carolina Medical Center ${ }^{4}$. Independientemente del porcentaje exacto de complicaciones, lo cierto es que por lo general se trata de grandes incisiones con sección de gran cantidad de masa muscular lo cual habitualmente va a suponer un prolongado tiempo de recuperación e incorporación a la actividad diaria del paciente.

Este panorama cambia radicalmente en 1992 con la descripción del abordaje suprarrenal por técnica laparoscópica trans-abdominal realizado por Gagner ${ }^{5}$. En esta aplicación la laparoscopia adquiere probablemente su máxima expresión de cirugía mínimamente invasiva dado que para la extracción de una pieza por lo general pequeña la cirugía convencional recurría a grandes incisiones debido a la profundidad del campo en el que se trabaja; como ejemplo de esto, en el $4^{\circ}$ volumen de la $8^{\underline{a}}$ edición del tratado de Urología de Campbell (2002), en el capítulo dedicado a la cirugía de las glándulas suprarrenales, recomienda el "uso de frontoscopio con fuente de luz para obtener una visualización correcta"6 (Fig. 2).

En el caso de nuestro Hospital, como ya se ha descrito en otros artículos, la laparoscopia retroperitoneal irrumpe en Junio de 2004 y tras casi tres años de realizar laparoscopia pélvica (prostatectomía radical). Se contactó con los diferentes servicios que manejan habitualmente la patología suprarrenal en nuestro centro (Endocrinología,

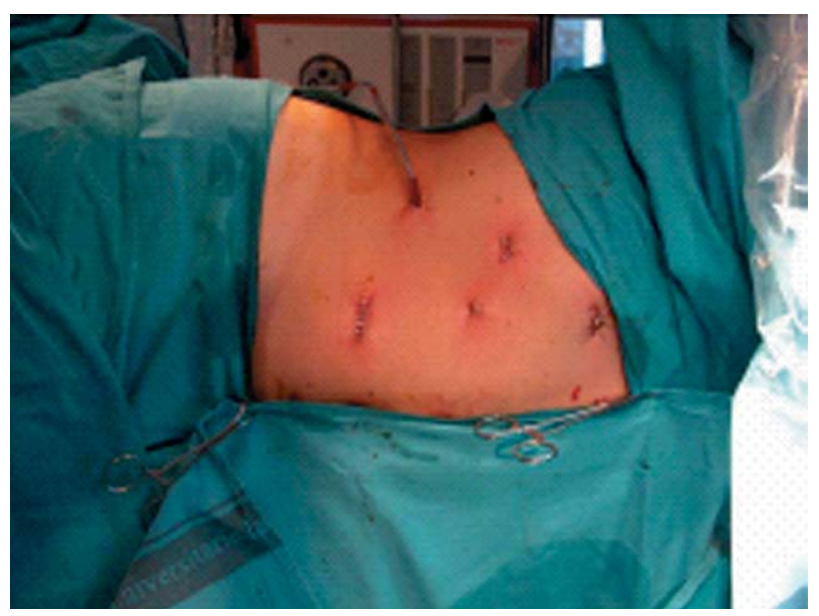

FIGURA 2. Incisiones laparoscópicas. 
Medicina Interna, Nefrología y Oncología por la posibilidad de lesiones metastásicas). El servicio de Cirugía General dispone de una sección de cirugía endocrinológica que realiza dicha intervención por vía abierta. La primera adrenalectomía se realizó en un paciente remitido de Medicina Interna por un posible sindrome de Conn secundario a un adenoma izquierdo y se realizó en noviembre de 2004 cuando ya se llevaban realizadas 21 intervenciones retroperitoneales laparoscópicas. En este artículo analizaremos nuestra experiencia inicial en cirugía adrenal laparoscópica y revisaremos la serie de los 10 últimos años de suprarrenalectomía abierta de este Servicio.

\section{MATERIAL Y MÉTODOS}

El comienzo del programa de cirugía laparoscópica retroperitoneal nos ha llevado a comparar la serie de suprarrenalectomías laparoscópicas frente a aquellas realizadas en los 10 años anteriores por vía abierta. El periodo de cirugía abierta abarcado es el que va desde enero 1994 hasta noviembre de 2004 (fecha de la realización de la primera adrenalectomía laparoscópica en nuestro servicio). Un total de 34 suprarrenalectomías han sido realizadas durante este periodo (24 por cirugía abierta y 10 por laparoscopia).

La técnica laparoscópica empleada ha sido el abordaje transperitoneal en 9 casos y sólo en uno se realizó un abordaje por lumboscopia debido a los antecedentes quirúrgicos del paciente (suprarrenalectomía derecha en paciente intervenido de quiste hepático hidatídico y posterior corrección de eventración con malla).

Como contraindicaciones absolutas laparoscópicas se han considerado la sospecha radiológica de infiltración periadrenal y el embarazo. A todos los pacientes se les realizó un TAC o RNM y se hizo un estudio para descartar la existencia de feocromocitoma. Inicialmente se descartó la cirugía del feocromocitoma ya que considerábamos que nuestra corta experiencia en dicha técnica nos llevaría a una mayor manipulación de la pieza con el riesgo de liberación de catecolaminas que esto supondría. Posteriormente, tras 76 intervenciones retroperitoneales, se realizó la primera adrenalectomía por feocromocitoma con éxito. Las indicaciones quirúrgicas han sido las masas funcionantes de cualquier tamaño y las masas afuncionantes sólidas de más de $5 \mathrm{~cm}$ de diámetro (Figs. 3 y 4).

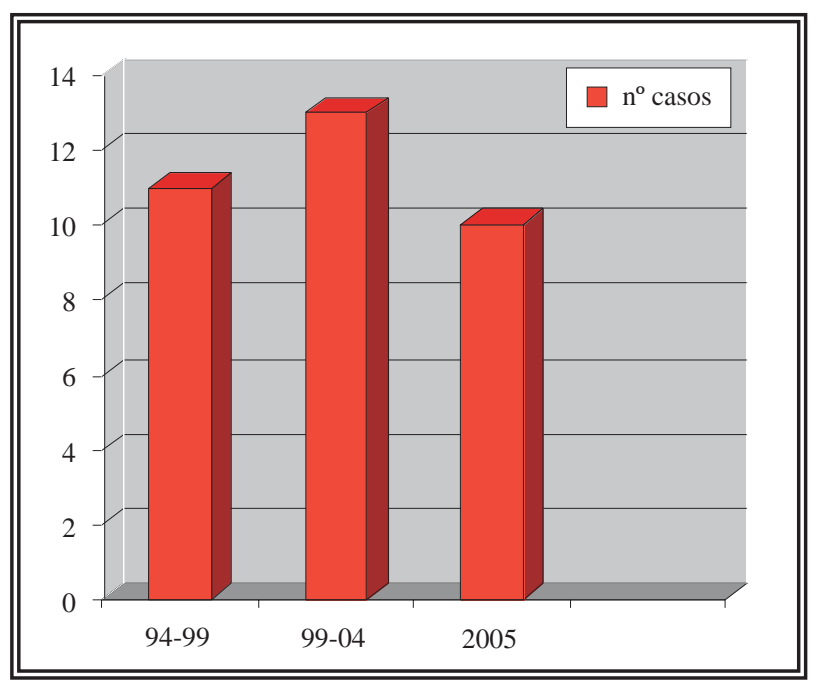

FIGURA 3. Número de casos por años.

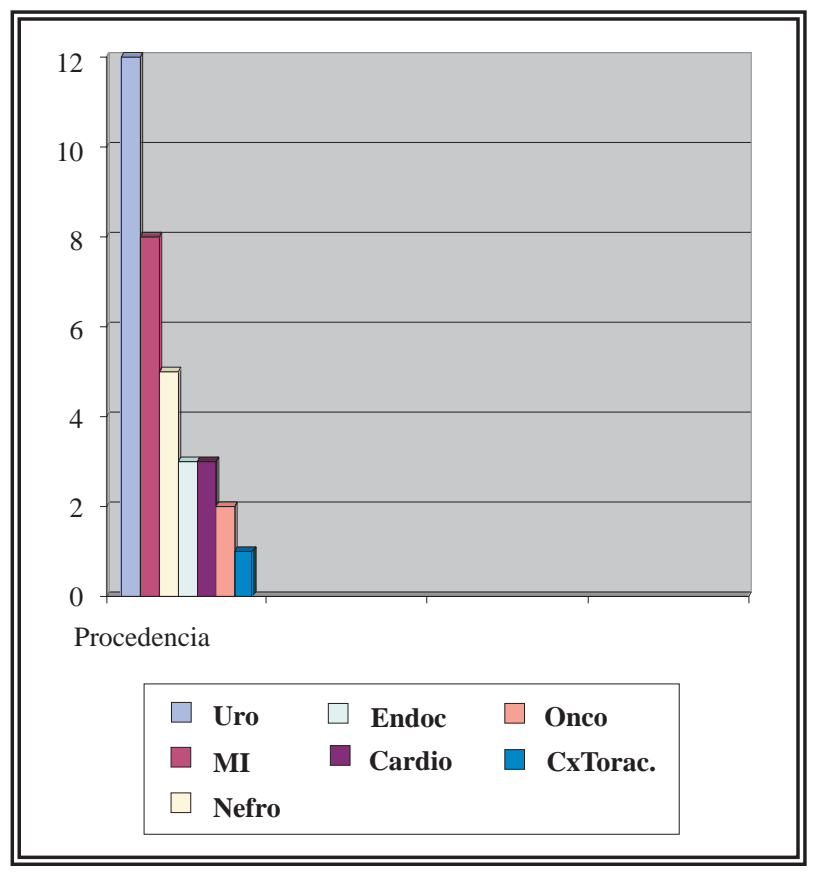

FIGURA 4. Servicios de procedencia.

\section{RESULTADOS}

Desde 1994 hasta 2005 el número de suprarrenalectomías realizadas en nuestro servicio fue de 34 casos, de las cuales 10 se realizaron en el último año por vía laparoscópica (29\%). De la serie de cirugía convencional, 19 casos eran varones $(79 \%)$ y 5 mujeres (21\%), con una edad media 
de la serie de 52,4 años (21-77) y una estancia media es de 7,25 días (3-13). En 7 casos de los 24 (29\%) existían antecedentes de cirugía abdominal o retroperitoneal. En 15 pacientes se realizaron abordajes derechos, en 8 izquierdos y en 1 caso la suprarrenalectomía fue bilateral. Un caso requirió reintervención por sangrado así como transfusión, hubo 2 pacientes con derrames pleurales que requirieron tubo de tórax, 1 íleo paralítico (caso $\mathrm{n}^{\circ} 19$ feocromocitoma bilateral con incisión subcostal bilateral) y un seroma importante de la herida. La anatomía patológica demostró un feocromocitoma en 6 ocasiones, un adenoma en 5 casos, 1 carcinoma suprarrenal en 4, dos hiperplasias, en 3 ocasiones una metástasis contralateral de un carcinoma renal, 1 tumor fibroso solitario, 1 hemorragia suprarrenal, 1 metástasis enteroide y 1 mielolipoma. El servicio de procedencia fue la propia consulta de Urologia en 10 ocasiones (41\%), Medicina Interna en 7 , Cardiología en 3 casos, Nefrología en 2 pacientes y Oncología y Cirugía Torácica en 1 caso cada una.

De la serie laparoscópica (noviembre de 2004 hasta diciembre de 2005), 7 eran varones y 3 mujeres, la edad media es de 49,6 años (35-79) y la estancia media es de 2,3 días (2-4). En 3 casos (30\%) existían antecedentes de cirugía abdominal o retroperitoneal previa (colecistectomía, quiste hidatídico, hemicolectomía, apendicectomía con peritonitis). El tiempo medio quirúrgico fue de 108 minutos (60-180), siendo el más prolongado el correspondiente a la suprarrenal realizada por lumboscopia. En 4 ocasiones la suprarrenalectomía fue izquierda y en 6 derecha. Ningún caso requirió transfusión. Se produjo un exitus en el postoperatorio del caso $\mathrm{n}^{-} 9$ por accidente cerebrovascular; se trataba de una paciente de 79 años, ASA 3, con antecedentes de accidentes isquémicos transitorios, remitida por el servicio de Endocrinología por presentar un adenoma funcionante de suprarrenal derecha que le ocasionaba un sindrome de Cushing. La cirugía se realizó en 70 minutos sin incidencias y la paciente presentó un accidente cerebral tromboembólico masivo del hemisferio izquierdo a las 12 horas de la intervención. La familia no autorizó la realización de la autopsia. En cuanto a los resultados de Anatomía Patológica, en 5 ocasio- nes se trataba de un adenoma, 2 hiperplasias, 1 metástasis de un carcinoma de colon, 1 mielolipoma y 1 feocromocitoma. En cuanto a los servicios de procedencia, Nefrología y Endocrinología son los que más casos han enviado, 3 cada uno de ellos; las consultas de Urología han remitido 2 casos y Medicina Interna y Oncología 1 caso cada una.

\section{DISCUSIÓN}

La técnica laparoscópica se ha convertido en el gold Standard de la patología adrenal. Múltiples estudios han demostrado sus ventajas: menor estancia hospitalaria, menor empleo de analgesia, más rápida recuperación, menor morbilidad $^{7-11}$.

Pero no nos engañemos, esta técnica requiere un aprendizaje prolongado y debemos tener en mente la posibilidad de un accidente vascular de difícil solución laparoscópica. En el caso concreto de la suprarrenalectomía derecha, su situación laterocava y la vena suprarrenal habitualmente corta y apical hace que debamos extremar las precauciones (Fig. 5). En este sentido, generalmente nos ayudamos de un trócar accesorio para levantar el hígado. La identificación de la vena renal principal y una buena liberación de la cava en su cara anterior y lateral es mandatorio. El campo quirúrgico de la suprarrenal izquierda habitualmente presenta menor compromiso vascular y de espacio. La correcta preparación del campo requiere la movilización completa del bazo y de los ligamentos lienorrenales, así como del mesocolon y la cola del páncreas. Estas referen-

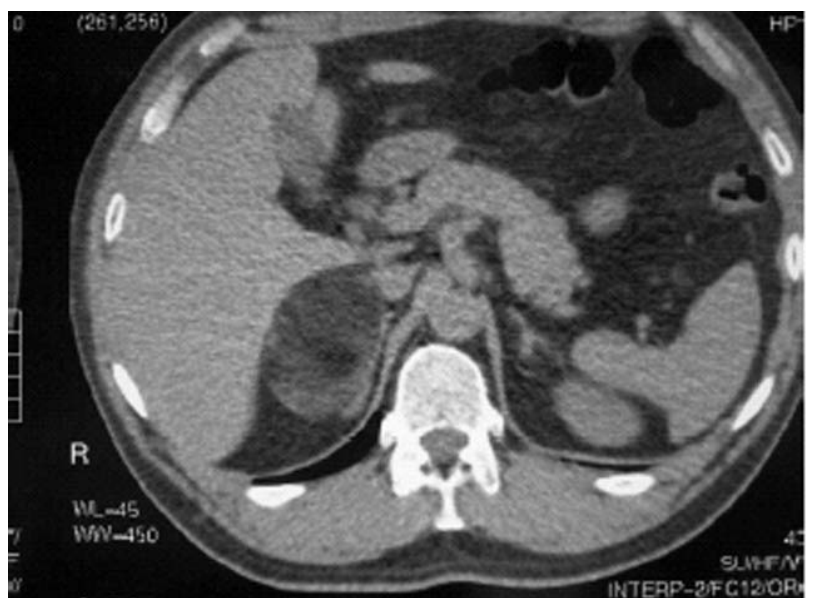

FIGURA 5. Mielolipoma de suprarrenal derecha. 
cias las utilizamos para la técnica transperitoneal, en nuestro Servicio la experiencia con lumboscopia es mucho menor.

Además de la dificultad técnica hay que añadir la todavía polémica indicación en alguna patología adrenal como puede ser el feocromocitoma o el carcinoma suprarrenal.

En el caso del feocromocitoma, la duda surge principalmente ante la posibilidad de que el incremento de la presión intraabdominal con la generación del neumoperitoneo desencadene una liberación de catecolaminas por parte del tumor superior a la que se podría desencadenar con la cirugía abierta, lo cual pondría en peligro la vida del paciente ${ }^{12-15}$.

El estímulo para la secreción de catecolaminas puede deberse a la presión sobre la glándula, cambios en la vascularización de la glándula o a la hipercapnia producidos por el neumoperitoneo ${ }^{16,17}$. Posteriormente a la inducción del neumoperitoneo, la manipulación de la glándula podría provocar una secreción de catecolaminas de magnitud similar a la cirugía abierta ${ }^{18}$. Para algunos cirujanos el traumatismo durante la disección es menor con la laparoscopia, probablemente debido a una mejor visión del campo quirúrgico y por lo tanto una disección más delicada con menor manipulación de la glándula ${ }^{10}$. Otros autores han observado una menor secreción de catecolaminas con la laparoscopia ${ }^{19}$.

Si bien inicialmente la indicación de la exéresis laparoscópica del feocromocitoma se encontraba en entredicho, son cada vez más las series que describen dicha técnica como segura y eficaz en manos de cirujanos con experiencia ${ }^{20,21}$.

La otra indicación controvertida en la aplicación de la laparoscopia es la suprarrenalectomía en patología maligna, metastásica o primaria. La posibilidad de metástasis en puertos o de diseminación peritoneal en los abordajes anteriores ha frenado su aplicación. Desde el primer caso descrito de metástasis en puertos en 1978 por una laparoscopia diagnóstica en un tumor de ovario se han descrito más casos en la literatura, apareciendo la primera referencia urológica en 1994 por una linfadenectomía de un carcinoma vesical de alto grado en la cual no se empleó bolsa de extracción ${ }^{23}$. En una revisión de 10.912 procedimientos laparoscópicos urológicos por cáncer, se registraron 13 casos de implantes tumorales $(0,1 \%)$, en 10 de ellos se localizaron en los puertos $(0,09 \%)$ y en 3 casos en peritoneo $(0,03 \%)$. Del total de 336 adrenalectomías, 3 casos presentaron implantes en puertos y 1 en peritoneo $(0,9 \%)$. 3 eran metástasis de un carcinoma pulmonar (2 pT4G3 y 1 pT3G3) y 1 se trataba de un carcinoma adrenocortical que fue reconvertido a cirugía abierta por invasión hepática y de la cava. En uno de los casos no se empleó bolsa de extracción ${ }^{24}$.

En otra revisión sobre 1.098 intervenciones urológicas de las cuales sólo 11 eran adrenalectomías, se detectaron 8 recurrencias en puertos de las cuales 1 correspondía a una suprarrenalectomía por una metástasis de un melanoma ${ }^{25}$. Existen más casos descritos en la literatura de implantes tumorales en los puertos de trabajo, sobre todo en intervenciones de Cirugía General y Ginecología 26,27 .

No está claro el mecanismo responsable de la metástasis en puertos, aunque todo parece indicar que se debe tratar de un proceso multifactorial en el cual el grado de experiencia del cirujano juega un papel importante. Otros factores relacionados con este proceso podrían ser el grado tumoral, rotura del tumor, contaminación del instrumental, neumoperitoneo, efecto chimenea de los trócares, $\operatorname{etc}^{28} \ldots$.

Como hemos indicado anteriormente, en este artículo presentamos nuestra experiencia inicial en cirugía adrenal laparoscópica. No se trata de una serie extensa ya que dicha patología se encuentra muy dispersa en nuestro hospital y así lo demuestra el bajo número de casos realizados en 10 años, pero lo que tratamos de transmitir es cómo iniciamos un programa de estas características, qué criterios quirúrgicos seguimos y los buenos resultados obtenidos en este periodo; precisamente son estos resultados los que nos animan a seguir con el proyecto. Llama la atención el hecho de que se han realizado en un año 10 cirugías frente a las 24 realizadas en los 10 años anteriores; en este sentido es fundamental el estrecho trato con los especialistas de donde proceden los pacientes (tanto pre como postoperatoriamente) y el hecho de que el Servicio de Cirugía General no realice adrenalectomías laparoscópicas. La técnica que creemos de elección en 
los comienzos es el abordaje transperitoneal. En cuanto a las contraindicaciones, parece lógico mantener como absoluta la sospecha de carcinoma suprarrenal con infiltración local. El feocromocitoma y el carcinoma suprarrenal localizado si creemos que pueden ser abordados mediante cirugía laparoscópica teniendo cierta experiencia.

\section{REFERENCIAS}

1. Saunders BD, Doherty GM. Laparoscopic adrenalectomy for malignant disease. Lancet Oncol. 2004 Dec;5(12):718726.

2. Saunder BD, Wainess RM, Dimick JB, Doherty GM, Upchurch GR, Gauger PG. Ann Arbor University, Michigan. Who performs endocrine operations in the United States? Surgery. 2003;134(6):924-931.

3. Thompson NW. The evolution of endocrine surgery as a subspecialty of general surgery. Fragmentation or enhancement? Arch Surg. 1996;131(5):465-471.

4. Cobb W, Kercher KW, Sing RF, Heniford B. Laparoscopic adrenalectomy for malignancy. The American Journal of Surgery. 2005:189(4):405-411

5. Gagner M, Lacroix A, Bolte E. Laparoscopic adrenalectomy in Cushing's syndrome and pheochromocytoma. N Engl J Med 1992;(14)327:1033.

6. Las glándulas suprarrenales. Campbell 8ª ed, vol 4:3894.

7. Duh QY, Siperstein AE, Clark OH, Steinberg AP, Meraney. Laparoscopic adrenalectomy.Comparison of the lateral and posterior approaches. Arch Surg 1996;131(8):870-876.

8. Smith CD, Weber CJ, Amerson JR. Laparoscopic adrenalectomy: new gold standard. World J Surg 1999;23(12): 389-396.

9. Dudley NE, Harrison BJ. Comparison of open posterior versus transperitoneal laparoscopic adrenalectomy. $\mathrm{Br} J$ Surg. 1999;86(5):656-660.

10. Thompson GB, Grant CS, Van Heerden JA. Laparoscopic versus open posterior adrenalectomy: a case-control study of 100 patients. Surgery. 1997;122(6):1132-1136.

11. Imai T, Kikumori T, Ohiwa Ml. A case-controlled study of laparoscopic compared with open lateral adrenalectomy. Am J Surg. 1999;178(1)50-53.

12. Colson P, Ribstein J. Simplified strategy for anesthesia of pheochromocytoma. Ann Fr Anesth Reanim. 1991;10(5): 456-462.

13. Col V, de Canniere L, Collard E, Michel L, Donckier J. Laparoscopic adrenalectomy for phaeochromocytoma: endocrinological and surgical aspects of a new therapeutic approach. Clin Endocrinol. (Oxf) 1999;50(1):121-125.

14. Joris JL, Hamoir EE, Hartstein GM, Meurisse MR, Hubert BM, Charlier CJ. Hemodynamic changes and catecholamine release during laparoscopic adrenalectomy for pheochromocytoma. Anesth Analg. 1999;88(1):16-21.
15. Janetschek G, Neumann HP. Laparoscopic surgery for pheochromocytoma. Urol Clin North Am. 2001;28(1):97-105.

16. De La Chapelle A, Deghmani M,Dureuil B. Peritoneal insufflation can be a critical moment in the laparoscopic surgery of pheochromocytoma. Ann Fr Anesth Reanim. 1998;17(9): 1184-1185.

17. Mann C, Millat B, Boccara G, Atger J, Colson P. Tolerance of laparoscopy for resection of phaeochromocytoma. Br J Anaesth. 1996;77(6):795-797.

18. Joris JL, Hamoir EE, Hartstein GM, Meurisse MR, Hubert BM, Charlier CJ. Hemodynamic changes and catecholamine release during laparoscopic adrenalectomy for pheochromocytoma. Anesth Analg. 1999;88(1):16-21.

19. Fernández-Cruz L, Saenz A, Taura P, Benarroch G, Nies C, Astudillo E. Pheochromocytoma: laparoscopic approach with $\mathrm{CO} 2$ and helium pneumoperitoneum. Endosc Surg Allied Technol. 1994;2(6):300-304.

20. Dennis L. Fowler, MD. Laparoscopic Adrenalectomy: There Can Be No Doubt. Annals of Surgical Oncology. 10(9):997998 Editorial.

21. Flavio Rocha M, Faramarzi-Roques R, Tauzin-Fin P, Vallee V, Leitao de Vasconcelos PR, Ballanger P. Laparoscopic Surgery for Pheochromocytoma. European Urology. 2004; 45(2):226-232.

22. Mobius E, Nies C, Rothmund M. Surgical treatment of pheochromocytomas. Laparoscopic or conventional? Surg Endosc. 1999;13(1):35.

23. Stolla V, Rossi D .Subcutaneus metastasis after coelioscopic lymphadenectomy for vesical urothelial carcinoma. Eur Urol. 1994;26(4):342-343.

24. Micali S, Celia A, Bove P. Tumor seeding in urological laparoscopy: an international survey. J Urol. 2004;171(6pt1): 2151-2154.

25. Rassweiler J, Tsilvian A. Oncological safety of laparoscopic surgery for urological malignancy: experience with more than 1.000 operations. J Urol. 2003;Jun;169(6):2072-2075.

26. Cirocco WC, Schwartzman A, Golub RW. Abdominal wall recurrence after laparoscopic colectomy for colon cancer. Surgery. 1994;116(5):842-846.

27. Jacquet P, Averbach AM, Jacquet N. Abdominal wall metastasis and peritoneal carcinomatosis after laparoscopicassisted colectomy for colon cancer. Eur J Surg Oncol. 1995;21(5):568-570.

28. Tsivian, A, Sidi AA. Port site metastases in urological laparoscopic surgery. J Urol. 2003;169(4):1213.

Dr. A. Aguilera Bazán

E-mail: aaguilera11@yahoo.es

(Trabajo recibido el 3 de marzo de 2006) 